\title{
Gravitons in a Casimir box
}

\section{Francesco Alessio, $^{a, b}$ Glenn Barnich ${ }^{b}$ and Martin Bronte ${ }^{b}$}

${ }^{a}$ Dipartimento di Fisica "E. Pancini" and INFN Università degli studi di Napoli "Federico II", I-80125 Napoli, Italy

${ }^{b}$ Physique Théorique et Mathématique, Université libre de Bruxelles and International Solvay Institutes, Campus Plaine C.P. 231, B-1050 Bruxelles, Belgium

E-mail: francesco.alessio@unina.it, gbarnich@ulb.ac.be, Martin.Bonte@ulb.ac.be

ABSTRACT: The partition function of gravitons with Casimir-type boundary conditions is worked out. The simplest box that allows one to achieve full analytical control consists of a slab geometry with two infinite parallel planes separated by a distance $d$. In this setting, linearized gravity, like electromagnetism, is equivalent to two free massless scalar fields, one with Dirichlet and one with Neumann boundary conditions, which in turn may be combined into a single massless scalar with periodic boundary conditions on an interval of length $2 d$. When turning on a chemical potential for suitably adapted spin angular momentum, the partition function is modular covariant and expressed in terms of an Eisenstein series. It coincides with that for photons. At high temperature, the result provides in closed form all sub-leading finite-size corrections to the standard (gravitational) black body result. More interesting is the low-temperature/small distance expansion where the leading contribution to the partition function is linear in inverse temperature and given in terms of the Casimir energy of the system, whereas the leading contribution to the entropy is proportional to the area and originates from gravitons propagating parallel to the plates.

KEYWords: Discrete Symmetries, Models of Quantum Gravity, Thermal Field Theory

ARXIV EPRINT: 2011.14432 


\section{Contents}

1 Introduction $\quad 2$

2 Casimir boundary conditions and mode expansions 3

3 Reduced phase space sectors $\quad 4$

3.1 Particle sector 4

3.2 Adapted polarization tensors 5

$\begin{array}{lll}3.3 & \text { Modes propagating parallel to the plates } & 6\end{array}$

$\begin{array}{lll}3.4 & \text { Modes with a normal component of propagation } & 7\end{array}$

4 Scalar field formulations $\quad 7$

$\begin{array}{lll}4.1 & \text { Bromwich-Borgnis fields } & 7\end{array}$

4.2 Additional observable 8

$\begin{array}{llr}4.3 \text { Single scalar field formulation } & 10\end{array}$

5 Partition function $\quad \mathbf{1 0}$

$\begin{array}{lll}\text { 5.1 Real analytic Eisenstein series from functional approach } & 10\end{array}$

$\begin{array}{ll}5.2 \text { Low and high temperature expansions from canonical approach } & 11\end{array}$

$\begin{array}{ll}5.3 & \text { Complex trigonometric functions from method of images } \\ \end{array}$

$\begin{array}{lll}6 & \text { Discussion } & 13\end{array}$

$\begin{array}{ll}\text { A Photons in a Casimir box revisited } & 14\end{array}$

$\begin{array}{ll}\text { A.1 Boundary conditions and mode decomposition } & 14\end{array}$

$\begin{array}{lll}\text { A.2 The particle } & 15\end{array}$

$\begin{array}{lll}\text { A.3 Polarization vectors } & 15\end{array}$

$\begin{array}{lll}\text { A.4 Adapted polarization vectors } & 16\end{array}$

$\begin{array}{lll}\text { A.5 Bromwich-Borgnis fields } & 18\end{array}$

$\begin{array}{lll}\text { A.6 Single scalar field formulation } & 18\end{array}$

$\begin{array}{lll}\text { A.7 Partition function of photons } & 19\end{array}$

B Linearized gravity in empty space $\quad 19$

$\begin{array}{ll}\text { B.1 Hamiltonian formulation of Pauli-Fierz theory } & 19\end{array}$

B.2 Polarization tensors 20

C Generalized curl and projection operators on modes 21 


\section{Introduction}

What boundary conditions may be consistently imposed on gauge and gravitational fields, both asymptotically or at finite distances, is an important problem that has recently attracted a lot of attention because it is directly related not only to asymptotic and residual gauge symmetries but also to the number and the nature of the degrees of freedom of the system.

In his report on quantum fields in curved space ([1], section 2.4), DeWitt explains that we know "from years of experiment and years of model building" what a conductor is and what consistent boundary conditions to impose for electric and magnetic fields. He then goes to infer meaningful boundary conditions and boundary terms for the case of a massless scalar field.

In this work, we follow the spirit of DeWitt's approach and impose the analog of "perfectly conducting" boundary conditions on free massless spin 2 fields. We will show that the partition function of gravitons in such a Casimir box can be computed in a straightforward way and is identical to that of photons in the original Casimir set-up, and also to that of a massless scalar field with periodic boundary conditions in a box twice as big. This is not entirely surprising since these are results on the level of free field theories.

At this stage, we will limit ourselves to deriving the exact analytic and modular covariant result that allows one to access the qualitatively very different high and low temperature expansions, in agreement with recent results on a Cardy formula and modular properties in higher dimensions [2-4]. We will not address whether one may in principle confine gravitons to a box so as to achieve thermal equilibrium [5-8]. Neither will we speculate on the physical meaning of perfect conductors for gravitons nor comment on the relation to a recent study of the gravitational Casimir effect at zero temperature with non-idealized boundary conditions [9]. As compared to the closely related computations in [10], where a spherical box as a model for a Schwarzschild black hole is considered, the use of a slab geometry simplifies a detailed discussion of the contribution of various degrees of freedom to the partition function and thus to the entropy, in much the same way than such a geometry makes computations and conceptual issues relatively straightforward in the original Casimir analysis [11], whereas the spherically symmetric case [12, 13] is much more involved. Finally, we will not discuss here of how such computations in linearized gravity could be relevant for black hole physics in full non-linear general relativity or compare to results in the context of cosmological models [14].

The paper is organized as follows. As a useful preparation, we re-discuss in appendix A, our analysis $[15,16]$ of photons with Casimir boundary conditions by using polarization vectors adapted to $E$ and $H$ modes. We also briefly review in appendix B the well-known reduced phase space of linearized gravity in empty space. In the main text, we start in section 2, by defining Casimir-type boundary conditions with the associated mode expansions for metric perturbations and their canonical momenta. This allows us in section 3 to work out the physical degrees of freedom in this precise set-up and to isolate the sector with vanishing transverse momentum, or in other words, the sector of gravitational waves propagating parallel to the plates. The next step in section 4 consists in organizing all 
physical degrees of freedom, first in terms of two massless scalar fields with Dirichlet and Neumann boundary conditions, respectively and then in terms of a single scalar field. In order to do so, we follow recent work on the finite temperature electromagnetic Casimir effect and modular invariance in this context [17] to show that this scalar field should satisfy periodic boundary on an interval of double the length of the separation of the plates. This allows us in section 5 to simply infer the exact result for the partition function for gravitons, with a chemical potential for suitably modified spin angular momentum turned on, from the well-studied case of a massless scalar field with linear momentum in the compact direction turned on $[2-4,18]$, together with modular properties and consistent high and low temperature expansions.

Arguably the most interesting aspect of the exact analytic result is the fact that the low temperature/small box expansion is qualitatively very different from the more standard high temperature/large volume expansion. The latter is dominated by the standard black body result, up to finite size corrections. At low temperature however, the leading contribution to the partition function scales like inverse temperature and is responsible for the zero temperature gravitational Casimir force. It does not contribute to the entropy. The sub-leading contribution to the partition function is then the leading contribution to the entropy. It is proportional to the area of the plates and our analysis shows that it originates from gravitons that propagate parallel to those plates. The dynamics of those gravitons is equivalent to that of photons in two spatial dimensions, which in turn is described by a massless scalar field in 2 spatial dimensions. Let us stress here that these degrees of freedom are due to the non-trivial boundary conditions imposed on the fields but, except for the particle present in the spectrum, there is ultimately no interpretation in terms of residual gauge degrees of freedom nor a direct relation to large gauge transformations, contrary to what is implied in $[15,16]$.

\section{Casimir boundary conditions and mode expansions}

We want to promote the empty space analysis of massless spin 2 fields in the Hamiltonian approach, as reviewed in appendix B, to a slab geometry with two infinite parallel planes at $x^{3}=-\frac{d}{2}$ and $x^{3}=\frac{d}{2}$. Let $a=1,2, i=a, 3, V=L_{1} L_{2} d$ the volume of the system with $L_{a}$ taken large in the end, and $d$ the separation of the plates. Let $k_{3}=\frac{\pi n_{3}}{d}, k_{a}=\frac{2 \pi n_{a}}{L_{a}}$ (with no summation over $a$ ).

In analogy with the electromagnetic case reviewed in appendix A, we define Casimirtype boundary conditions by requiring that $h_{a b}, \pi^{a b}$ and $h_{33}, \pi^{33}$ satisfy Dirichlet conditions, while $h_{a 3}, \pi^{a 3}$ satisfy Neumann conditions. More explicitly, at the boundary $x^{3}=-\frac{d}{2}$ and $x^{3}=\frac{d}{2}$, we require

$$
h_{a b}=0=\pi^{a b}, \quad h_{33}=0=\pi^{33}, \quad\left(\partial_{3} h_{a 3}\right)=0=\left(\partial_{3} \pi^{a 3}\right) .
$$

Let us use $T^{i j}$ for either $h_{i j}$ or $\pi^{i j}$ and define, as in (A.2), a complete set of scalar functions $\psi_{k}^{H}$ with $n_{a}, n_{3}>0$ adapted to Dirichlet boundary conditions, and $\psi_{k}^{E}$ with $n_{a}, n_{3} \geq 0$ adapted to Neumann boundary conditions. 
The boundary conditions on the canonically conjugate variables are thus implemented through the mode expansions ${ }^{1}$

$$
T^{a b}(x)=\sum_{n_{a}, n_{3}>0} T_{k}^{a b} \psi_{k}^{H}, \quad T^{33}(x)=\sum_{n_{a}, n_{3}>0} T_{k}^{33} \psi_{k}^{H}, \quad T^{a 3}(x)=(-i) \sum_{n_{a}, n_{3} \geq 0} T_{k}^{a 3} \psi_{k}^{E},
$$

where we take $T_{k_{a}, 0}^{a b}=0=T_{k_{a}, 0}^{33}$. Reality conditions are

$$
T_{k_{a}, k_{3}}^{a b}=T_{-k_{a}, k_{3}}^{* a b}, \quad T_{k_{a}, k_{3}}^{33}=T_{-k_{a}, k_{3}}^{* 33}, \quad T_{k_{a}, k_{3}}^{a 3}=-T_{-k_{a}, k_{3}}^{* a 3},
$$

while parity conditions are

$$
T_{k_{a}, k_{3}}^{a b}=-T_{k_{a},-k_{3}}^{a b}, \quad T_{k_{a}, k_{3}}^{33}=-T_{k_{a},-k_{3}}^{33}, \quad T_{k_{a}, k_{3}}^{a 3}=T_{k_{a},-k_{3}}^{a 3} .
$$

Odd variables do not have modes at $n_{i}=0, T_{0,0}^{a b}=0=T_{0,0}^{33}$, but there are two such modes in the even sector, $T_{0,0}^{a 3} \neq 0$.

Note that these boundary conditions have been constructed in such a way that the left hand sides of the constraints have well defined expansions, either in terms of sines or in terms of cosines. If one requires the lapse and the shift to impose all constraints in a first order variational principle and not to introduce spurious variables, it follows from taking into account the mode expansion of the constraints (B.3), that the lapse $n$ and $n^{a}$ should satisfy Dirichlet while $n^{3}$ should satisfy Neumann conditions,

$$
n=\sum_{n_{a}, n_{3}>0} n_{k} \psi_{k}^{H}, \quad n^{a}=\sum_{n_{a}, n_{3}>0} n_{k}^{a} \psi_{k}^{H}, \quad n^{3}=\sum_{n_{a}, n_{3} \geq 0}^{\prime} n_{k}^{3} \psi_{k}^{E},
$$

so that $n, n^{a}$ are odd, while $n^{3}$ is even under $k_{3} \rightarrow-k_{3}$.

\section{$3 \quad$ Reduced phase space sectors}

In terms of these decomposition, the kinetic term is given by

$$
\int \mathrm{d}^{3} x \partial_{0} h_{i j} \pi^{i j}=\sum_{n_{a}, n_{3} \geq 0} \partial_{0} h_{i j} \pi^{* i j}
$$

where we sometimes omit the $k$ dependence of the Fourier coefficients for notational simplicity. The Pauli-Fierz Hamiltonian (B.2) is given by

$$
H_{\mathrm{PF}}=\sum_{n_{a}, n_{3} \geq 0}\left[\pi^{i j} \pi_{i j}^{*}-\frac{1}{2} \pi \pi^{*}+\frac{1}{4} k^{2} h_{i j} h^{* i j}-\frac{1}{2} k_{i} h^{i j} k^{l} h_{l j}^{*}+\frac{1}{2} k^{i} k^{j} h h_{i j}^{*}-\frac{1}{4} k^{2} h h^{*}\right] .
$$

\subsection{Particle sector}

We start with the particle sector, which has to be treated separately since the change of variables that we will use below does not apply to this sector. For $n_{i}=0$, we define $h_{a 3,0,0}=\sqrt{2} q^{a}$ and $\pi_{0,0}^{a 3}=\frac{1}{\sqrt{2}} p_{a}$. From the expansion of the kinetic term, these variables are canonically conjugate. None of these variables is involved in the constraints nor affected by a gauge transformation generated by these constraints. Their contribution to the PauliFierz Hamiltonian is that of two particles of unit mass,

$$
H_{n_{i}=0}=\frac{1}{2} p^{a} p_{a}
$$

\footnotetext{
${ }^{1}$ The factor $(-i)$ in front of the expansion of $\left(h_{a 3}, \pi^{a 3}\right)$ is chosen for later convenience.
} 


\subsection{Adapted polarization tensors}

For $n_{i} \neq 0$, one may use the polarization tensors as defined in (B.5)-(B.8) to decompose the components. Furthermore, it will turn out to be convenient to use the polarization tensors built out of the adapted polarization vectors $e_{\lambda}{ }^{i}, \lambda=(H, E)$ given in (A.13) rather than to use generic transverse vectors $e_{\alpha}{ }^{i}$.

As a consequence,

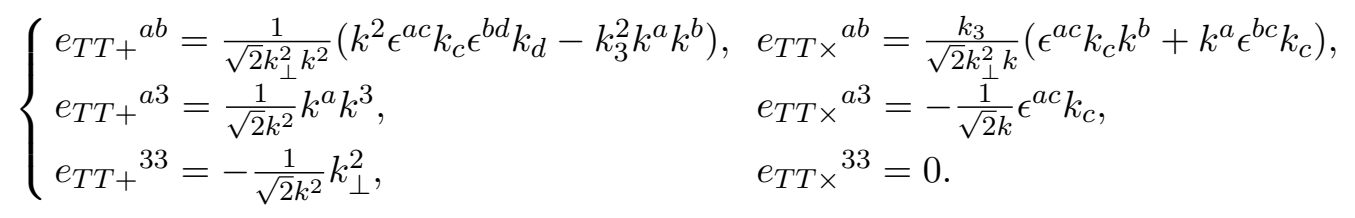

All variables now have the same reality conditions

$$
T_{k_{a}, k_{3}}^{T T s}=T_{-k_{a}, k_{3}}^{* T T s}, \quad T_{k_{a}, k_{3}}^{T}=T_{-k_{a}, k_{3}}^{* T}, \quad T_{k_{a}, k_{3}}^{L T \lambda}=T_{-k_{a}, k_{3}}^{* L T \lambda}, \quad T_{k_{a}, k_{3}}^{L L}=T_{-k_{a}, k_{3}}^{* L L},
$$

while parity conditions become

$$
\begin{array}{ll}
T_{k_{a}, k_{3}}^{T T \times}=T_{k_{a},-k_{3}}^{T T \times}, & T_{k_{a}, k_{3}}^{L T E}=T_{k_{a},-k_{3}}^{L T E}, \\
T_{k_{a}, k_{3}}^{T T+}=-T_{k_{a},-k_{3}}^{T T+}, & T_{k_{a}, k_{3}}^{T}=-T_{k_{a},-k_{3}}^{T}, \quad T_{k_{a}, k_{3}}^{L T H}=-T_{k_{a},-k_{3}}^{L T H}, \quad T_{k_{a}, k_{3}}^{L L}=-T_{k_{a},-k_{3}}^{L L} .
\end{array}
$$

If $\Xi=(T T s, T, L T \lambda, L L)$, the kinetic term is

$$
\int \mathrm{d}^{3} x \partial_{0} h_{i j} \pi^{i j=} \sum_{n_{a}, n_{3} \geq 0}^{\prime} \partial_{0} h_{\Xi} \pi^{* \Xi},
$$

so that the Poisson brackets are

$$
\left\{h_{\Xi k}, \pi_{k^{\prime}}^{* \Gamma}\right\}=\delta_{\Xi}^{\Gamma} \prod_{i=1}^{3} \delta_{n_{i}, n_{i}^{\prime}}
$$

The Hamiltonian constraints (B.3) become

$$
\begin{aligned}
\mathcal{H}_{a} & =-2 i \sum_{n_{a}, n_{3}>0}\left[k^{b} \pi_{a b}+k^{3} \pi_{a 3}\right] \psi_{k}^{H}=-2 i \sum_{n_{a}, n_{3}>0} k\left[e^{\lambda}{ }_{a} \frac{\pi_{L T \lambda}}{\sqrt{2}}+e^{\|_{a} \pi_{L L}}\right] \psi_{k}^{H}, \\
\mathcal{H}_{3} & =(-2) \sum_{n_{a}, n_{3} \geq 0}^{\prime}\left[k^{b} \pi_{b 3}+k^{3} \pi_{33}\right] \psi_{k}^{E}=-2 \sum_{n_{a}, n_{3} \geq 0}^{\prime} k\left[e^{\lambda}{ }_{3} \frac{\pi_{L T \lambda}}{\sqrt{2}}+e^{\|_{1}} \pi_{L L}\right] \psi_{k}^{E} \\
\mathcal{H}_{\perp} & =-\sum_{n_{a}, n_{3}>0} k^{2} \sqrt{2} h_{T} \psi_{k}^{H} .
\end{aligned}
$$

The Pauli-Fierz Hamiltonian (B.2) is given by

$$
H_{\mathrm{PF}}=\sum_{n_{a}, n_{3} \geq 0}^{\prime}\left[\pi^{T T s} \pi_{T T s}^{*}+\pi^{L T \lambda} \pi_{L T \lambda}^{*}+\frac{1}{2} \pi^{L L} \pi_{L L}^{*}+\frac{1}{4} k^{2}\left(h_{T T s} h^{* T T s}-h_{T} h^{* T}\right)\right] .
$$




\subsection{Modes propagating parallel to the plates}

For $n_{i} \neq 0$, let us start the analysis with the sector at $n_{3}=0$. It is useful to consider the dyad

$$
e_{\perp}^{b}\left(k_{a}, 0\right)=\frac{\epsilon^{b c} k_{c}}{k_{\perp}}, \quad e_{\|}^{c}\left(k_{a}, 0\right)=\frac{k^{c}}{k_{\perp}}
$$

in terms of which a two-dimensional vector $T_{k_{a}, 0}^{b}$ may be decomposed as

$$
T_{k_{a}, 0}^{b}=T_{k_{a}, 0}^{\perp} e_{\perp}^{b}\left(k_{a}, 0\right)+T_{k_{a}, 0}^{\|} e_{\|}^{b}\left(k_{a}, 0\right)
$$

When using $T_{k_{a}, 0}^{a b}=0=T_{k_{a}, 0}^{33}$ with only $T_{k_{a}, 0}^{a 3} \neq 0$, it is straightforward to check using the explicit form of the polarization vectors that

$$
\begin{aligned}
& T_{k_{a}, 0}^{L L}=0, \quad T_{k_{a}, 0}^{T}=0, \quad T_{k_{a}, 0}^{L T H}=0, \quad T_{k_{a}, 0}^{T T+}=0, \\
& T_{k_{a}, 0}^{L T E}=-\frac{2}{\sqrt{2}} T_{k_{a}, 0}^{\| 3}, \quad T_{k_{a}, 0}^{T T \times}=-\frac{2}{\sqrt{2}} T_{k_{a}, 0}^{\perp 3} .
\end{aligned}
$$

At $n_{3}=0, n_{a} \neq 0$, the constraints reduce to

$$
\pi_{k_{a}, 0}^{L T E}=0
$$

or equivalently to $\pi_{k_{a}, 0}^{\| 3}=0$. On the constraint surface, the contribution from the $n_{3}=0$ modes to the first order action Pauli-Fierz action then reduces to the contribution of the canonical pair

$$
h_{k_{a}, 0}^{T T \times}=-\frac{2}{\sqrt{2}} h_{k_{a}, 0}^{\perp 3}, \quad \pi_{k_{a}, 0}^{T T \times}=-\frac{2}{\sqrt{2}} \pi_{k_{a}, 0}^{\perp 3},
$$

with Hamiltonian

$$
H_{\mathrm{PF}, n_{3}=0} \approx \sum_{n_{a}}^{\prime}\left(\pi_{k_{a}, 0}^{T T \times} \pi_{k_{a}, 0}^{* T T \times}+\frac{1}{4} k_{\perp}^{2} h_{k_{a}, 0}^{T T \times} h_{k_{a}, 0}^{* T T \times}\right) .
$$

After the canonical transformation $\pi_{k_{a}, 0}^{T T \times} \rightarrow \frac{1}{\sqrt{2}} \pi_{k_{a}, 0}^{T T \times}, h_{k_{a}, 0}^{T T \times} \rightarrow \frac{1}{\sqrt{2}} h_{k_{a}, 0}^{T T \times}$, it is obvious that this collection of harmonic oscillators is the same than one would get from the expansion of a massless scalar field in 2 spatial dimensions with periodic boundary conditions (omitting its particle mode). That is the reason why we sometimes refer to this sector as the lower dimensional scalar field. This sector is somewhat unexpected when comparing to the empty space analysis in appendix B and is due to the perfectly conducting boundary conditions discussed in the previous section. From the explicit discussion of the structure of the constraints and the Hamiltonian, we have thus shown the following:

With Casimir-type boundary conditions (2.1), gravitational waves propagating parallel to the plates are equivalently described by electromagnetic waves in 2 spatial dimensions, whose physical degrees of freedom are in turn described by a massless scalar field in 2 spatial dimensions. 


\subsection{Modes with a normal component of propagation}

When $n_{3} \neq 0$, the constraints $\mathcal{H}_{i}=0$ in (3.9) are equivalent to

$$
e^{\lambda}{ }_{i} \frac{\pi_{L T \lambda}}{\sqrt{2}}+e^{\|_{i}} \pi_{L L}=0
$$

After contracting with $e_{A}^{i}$, the constraints (3.9) are then equivalent to the vanishing of specific components,

$$
\pi_{L T \lambda}=0=\pi_{L L}, \quad h_{T}=0 .
$$

On the constraint surface, the only variables that remain in the first order Hamiltonian action are $h_{T T s}, \pi^{T T s}$ with

$$
H_{\mathrm{PF}, n_{3}>0} \approx \sum_{n_{a}, n_{3}>0}\left(\pi_{T T s} \pi^{* T T s}+\frac{1}{4} k^{2} h_{T T s} h^{* T T s}\right) .
$$

Note that, if desired, one may also work with the gauge fixing conditions

$$
\triangle \pi-\partial_{i} \partial_{j} \pi^{i j}=0, \quad-2 \partial_{j} h^{i j}=0 .
$$

and consider Dirac brackets.

\section{Scalar field formulations}

\subsection{Bromwich-Borgnis fields}

Disregarding particle zero modes, the reduced phase space consists of two scalar fields and their momenta, one pair satisfying Dirichlet and the other Neumann boundary conditions,

$$
\begin{aligned}
h^{H} & =\sum_{n_{a}, n_{3}>0} h_{k}^{T T+} \psi_{k}^{H}, & \pi^{H} & =\sum_{n_{a}, n_{3}>0} \pi_{k}^{T T+} \psi_{k}^{H}, \\
h^{E} & =\sum_{n_{a}, n_{3} \geq 0}^{\prime} h_{k}^{T T \times} \psi_{k}^{E}, & \pi^{E} & =\sum_{n_{a}, n_{3} \geq 0}^{\prime} \pi_{k}^{T T \times} \psi_{k}^{E} .
\end{aligned}
$$

On account of the form of the Pauli-Fierz Hamiltonian, (3.19) and (3.16), there is thus a direct gravitational analogue of the electromagnetic results reviewed in appendix A.5:

With Casimir-type boundary conditions (2.1) the dynamics of physical gravitons is equivalent to that of two massless scalar fields, one with Dirichlet and one with Neumann boundary conditions, with action

$$
S\left[h^{\lambda}\right]=-\frac{1}{2} \int d^{4} x \partial_{\mu} h^{\lambda} \partial^{\mu} h_{\lambda}, \quad \lambda=(H, E) .
$$

Note that the massless scalar field in 2 spatial dimensions discussed in section 3.3 corresponds to the modes at $k_{3}=0$ contained in $h_{E}$.

The set-up follows closely the electromagnetic case discussed in appendix A, with the following substitutions:

$$
A_{k}^{E} \rightarrow \frac{1}{\sqrt{2}} h_{k}^{T T \times}, \quad \pi_{k}^{E} \rightarrow \sqrt{2} \pi_{k}^{T T \times}, \quad A_{k}^{H} \rightarrow \frac{1}{\sqrt{2}} h_{k}^{T T+}, \quad \pi_{k}^{H} \rightarrow \sqrt{2} \pi_{k}^{T T+} .
$$


This defines the oscillator variables $a_{k}^{\lambda}, a_{k}^{* \lambda}$ in (A.17) in terms of the Fourier components of the physical gravitational fluctuations with standard Poisson brackets (A.18). The Pauli-Fierz Hamiltonian without the particle contributions, given by the sum of the pieces in (3.16) and of (3.19), is equivalent to

$$
H_{\mathrm{PF}}^{\prime}=\sum_{\lambda, n_{a}, n_{3} \geq 0}^{\prime} \frac{1}{2}\left(\pi_{k}^{\lambda} \pi_{k}^{* \lambda}+k^{2} h_{k}^{\lambda} h_{k}^{* \lambda}\right)=\sum_{\lambda, n_{a}, n_{3} \geq 0}^{\prime} \frac{k}{2}\left(a_{k}^{\lambda} a_{k}^{* \lambda}+a_{k}^{* \lambda} a_{k}^{\lambda}\right) .
$$

Remark. In momentum space, the shift can be further expanded as $n_{k}^{i}=n_{k}^{\lambda} e_{\lambda}^{i}+n_{k}^{\|} e_{\|}^{i}$, with $n_{k}^{H}, n_{k}^{\|}$even and $n_{k}^{E}$ odd. In a variational principle with constraints included, variation with respect to these variables then directly impose the constraints (3.14) and (3.18). These variables may be lumped into fields

$$
n^{H, \|}=\sum_{n_{a}, n_{3}>0} n_{k}^{H, \|} \psi_{k}^{H}, \quad n^{E}=\sum_{n_{a}, n_{3} \geq 0}^{\prime} n_{k}^{E} \psi_{k}^{E}
$$

together with the expansion of the shift in (2.5).

In a Hamiltonian BFV-BRST approach, spatial diffeomorphism ghosts and the ghost associated to deformations normal to the surface that goes with the Hamiltonian constraint follow the same pattern, with the same expansion holding for their momenta. In an extended phase space, there are further canonically conjugate pairs of scalar fields,

$$
\begin{aligned}
\phi^{T, L T H, L L} & =\sum_{n_{a}, n_{3}>0} h_{k}^{T, L T H, L L} \psi_{k}^{H}, & \pi^{T, L T H, L L} & =\sum_{n_{a}, n_{3}>0} \pi_{k}^{T, L T H, L L} \psi_{k}^{H}, \\
\phi^{L T E} & =\sum_{n_{a}, n_{3} \geq 0}^{\prime} h_{k}^{L T E} \psi_{k}^{E}, & \pi^{L T E} & =\sum_{n_{a}, n_{3} \geq 0}^{\prime} \pi_{k}^{L T E} \psi_{k}^{E} .
\end{aligned}
$$

Quantization of all these variables should be done in terms of quartets so as to have achieve equivalence with the reduced phase space quantization described above. After integration over all momenta in the BRST gauge fixed path integral, this then gives rise to the BRST gauge fixed path integral in the Lagrangian approach, including the correct boundary conditions on all fields. Such an extended setting is useful in order to set up computations in covariant gauges (see e.g. [19]) for a review), and also when including interactions. We will not pursue this further here, but focus on reduced phase space quantization.

\subsection{Additional observable}

In order to achieve full modular covariance of the partition function below, an additional observable is of interest. Its expression is most transparent in terms of generalized vector calculus operations that feature prominently in the context of the Hamiltonian approach to duality invariance [20-24]. First, one considers the generalized vector product between a vector $v^{k}$ and a symmetric tensor $T_{i j}$

$$
(\vec{v} \times T)_{i j}=\frac{1}{2}\left(\epsilon_{i l m} v^{l} T_{j}^{m}+\epsilon_{j l m} v^{l} T_{i}^{m}\right) .
$$

This is then implies that the generalized curl for a symmetric spacetime tensor is,

$$
(\mathcal{O} T)_{i j}=(\vec{\nabla} \times T)_{i j}=\frac{1}{2}\left(\epsilon_{i l m} \partial^{l} T_{j}^{m}+\epsilon_{j l m} \partial^{l} T_{i}^{m}\right) .
$$


It rotates electric and magnetic components and projects out both the trace part $(T)$ and the longitudinal part of the longitudinal part $(L L)$. Then there is the operator that projects out the transverse components of the longitudinal part $(L T)$,

$$
(\mathcal{P} T)_{i j}=-\triangle T_{i j}+\partial_{i} \partial_{m} T_{j}^{m}+\partial_{j} \partial_{m} T_{i}^{m} .
$$

Combining these two operations gives a projector onto the transverse-traceless part,

$$
\left(\mathcal{P}^{T T} T\right)_{i j}=-(\mathcal{O P} T)_{i j}=-(\mathcal{P O} T)_{i j}
$$

Details on how these operator act are provided in appendix C.

For simplicity, we start by working in the reduced phase space with $T_{k}^{T}=T_{k}^{L T \lambda}=$ $T_{k}^{L L}=0$. In terms of space-time fields, we thus define $T_{T T}^{i j}$ through

$$
\begin{aligned}
& T_{T T}^{a b}=\sum_{n_{a}, n_{3}>0} e_{T T s}{ }^{a b} T_{k}^{T T s} \psi_{k}^{H}, \quad T_{T T}^{33}=\sum_{n_{a}, n_{3}>0} e_{T T s}{ }^{a b} T_{k}^{T T s} \psi_{k}^{H}, \\
& T_{T T}^{a 3}=-i \sum_{n_{a}, n_{3} \geq 0}^{\prime} e_{T T s}{ }^{a 3} T_{k}^{T T s} \psi_{k}^{E} .
\end{aligned}
$$

In analogy with (A.22), we then consider spin angular momentum in linearized gravity, defined on the reduced phase space by

$$
J_{i}=\int_{V} \mathrm{~d}^{3} x \pi_{T T}^{m n}\left(\vec{e}_{i} \times h^{T T}\right)_{m n},
$$

where $\vec{e}_{i}$ is the unit vector along $x^{i}$ in position space. When using that

$$
e_{T T+}{ }^{i j} e_{T T \times}{ }^{m n}-e_{T T \times}{ }^{i j} e_{T T+}{ }^{m n}=\frac{1}{2}\left[\epsilon^{i n l}\left(\delta^{j m}-\frac{k^{j} k^{m}}{k^{2}}\right)+\epsilon^{j m l}\left(\delta^{i n}-\frac{k^{i} k^{n}}{k^{2}}\right)\right] \frac{k_{l}}{k},
$$

which follows from (A.23), one finds in terms of modes that

$$
J_{3}=\sum_{n_{a}, n_{3}>0} \frac{k_{3}}{k}\left(h_{k}^{T T+} \pi_{k}^{* T T \times}-h_{k}^{T T \times} \pi_{k}^{* T T+}\right)=\sum_{n_{a}, n_{3}>0} \frac{i k_{3}}{k}\left(a_{k}^{* H} a_{k}^{E}-a_{k}^{* E} a_{k}^{H}\right) .
$$

The observable we are interested in is

$$
P_{3}^{S}=-\int_{V} \mathrm{~d}^{3} x \partial_{3}(\mathcal{O} h)_{m n} \frac{1}{\sqrt{-\triangle^{3}}}(\mathcal{P} \pi)^{m n},
$$

which is gauge invariant without the need to put unphysical components to zero by hand. When using the actions of $\mathcal{O}$ and $\mathcal{P}$ established in appendix $\mathrm{C}$, together with the orthonormality properties (B.9), it is straightforward to verify that its mode expansion differs from that of $J_{3}$ by multiplying each term in momentum space by $k$,

$$
P_{3}^{S}=\sum_{n_{a}, n_{3}>0} k_{3}\left(h_{k}^{T T+} \pi_{k}^{* T T \times}-h_{k}^{T T \times} \pi_{k}^{* T T+}\right)=\sum_{n_{a}, n_{3}>0} i k_{3}\left(a_{k}^{* H} a_{k}^{E}-a_{k}^{* E} a_{k}^{H}\right) .
$$




\subsection{Single scalar field formulation}

In the slab geometry considered here, one can go one step further and combine the Fourier components of the two scalar fields with Neumann and Dirichlet boundary into those of a single massless free scalar field with periodic boundary conditions on an interval of length $2 d$.

More precisely, from the expression of the Pauli-Fierz Hamiltonian (4.4) and the observable $P_{3}^{S}$ (4.16) in terms of modes and oscillators, one may show directly that these coincide with the standard Hamiltonian, omitting its particle mode, and linear momentum in the $x^{3}$ direction, of a massless scalar field with periodic boundary conditions in a rectangular box with sides $L_{1}, L_{2}, 2 d$. The proof is identical to that in the electromagnetic case reviewed in appendix A.6 when taking into account the identifications (4.3). In summary,

With Casimir-type boundary conditions (2.1), the dynamics of physical gravitons is equivalent to that of a massless scalar field with two large dimensions and with periodic boundary conditions on an interval of length $2 d$.

\section{Partition function}

From the above equivalence, the partition function for gravitons with Casimir-type boundary conditions may be obtained from that of a massless scalar field on $\mathbb{S}_{2 d}^{1} \times \mathbb{R}^{2}$. In turn, as shown in [17] and reviewed in appendix A, the same equivalence holds in the electromagnetic case. In the absence of a chemical potential, $\alpha=0$, up to a subtlety related to whether the black body result is subtracted or not, the result can thus also be obtained from the literature on the finite temperature electromagnetic Casimir effect $[25,26]$ (see also [27-35] and [36-38]) for reviews). It is however more economical to derive the result, whether the chemical potential is turned on or not, directly from the scalar field formulation. With $\alpha \neq 0$, this has been discussed instance in [2]. The detailed derivation by a variety of different methods of the expressions provided below can be found in [18].

\subsection{Real analytic Eisenstein series from functional approach}

The partition function

$$
Z(\beta, \alpha)=\operatorname{Tr} e^{-\beta \hat{H}+i \alpha \hat{P}_{3}^{S}},
$$

may be conveniently expressed in terms of the modular parameter defined by

$$
\tau=\frac{\alpha+i \beta}{L_{3}}, \quad L_{3}=2 d
$$

A further ingredient is the real analytic Eisenstein series,

$$
f_{2}(\tau, \bar{\tau})=\sum_{\left(n_{3}, n_{4}\right) \in \mathbb{Z}^{2} /(0,0)} \frac{\mathfrak{I m}(\tau)^{2}}{\left|n_{4}+n_{3} \tau\right|^{4}},
$$

which is invariant under modular transformations

$$
\tau^{\prime}=\frac{a \tau+b}{c \tau+d}, \quad a, b, c, d \in \mathbb{Z}, \quad a d-b c=1 .
$$


Starting from the Lagrangian path integral formulation and its relation to the appropriate zeta function, the partition function $\mathcal{Z}(\tau, \bar{\tau})=Z(\beta, \alpha)$ can be compactly written as

$$
\ln \mathcal{Z}(\tau, \bar{\tau})=\frac{1}{2 \pi^{2}} \frac{L_{1} L_{2}}{L_{3}^{2}} \frac{f_{2}(\tau, \bar{\tau})}{\mathfrak{I m}(\tau)}
$$

Since $f_{2}(\tau, \bar{\tau})$ is modular invariant and $\mathfrak{I m}(\tau)$ transforms as

$$
\mathfrak{I m}\left(\tau^{\prime}\right)=\frac{\mathfrak{I m}(\tau)}{|c \tau+d|^{2}}
$$

the partition function (5.5) transforms as

$$
\ln \mathcal{Z}\left(\tau^{\prime}, \bar{\tau}^{\prime}\right)=|c \tau+d|^{2} \ln \mathcal{Z}(\tau, \bar{\tau}) .
$$

If the chemical potential vanishes, $\alpha=0, \tau=i t$, with $t=\frac{\beta}{L_{3}}$ and $\mathrm{Z}(t)=\mathcal{Z}(i t,-i t)=$ $Z(\beta, 0)$, the result can be written in terms of an Epstein zeta function,

$$
\mathfrak{Z}\left(2 ; t^{2}, 1\right)=\sum_{n_{4}, n_{3}}^{\prime} \frac{1}{\left(n_{3}^{2} t^{2}+n_{4}^{2}\right)^{2}},
$$

as

$$
\ln \mathrm{Z}(t)=\frac{1}{2 \pi^{2}} \frac{L_{1} L_{2} t}{L_{3}^{2}} \mathfrak{Z}\left(2 ; t^{2}, 1\right) .
$$

If $a=0=d, b=1=-c$, the modular transformation reduces to temperature inversion $t \rightarrow \frac{1}{t}$ with

$$
\ln \mathrm{Z}\left(\frac{1}{t}\right)=t^{2} \ln \mathrm{Z}(t)
$$

\subsection{Low and high temperature expansions from canonical approach}

Let

$$
\xi(d)=\frac{\Gamma\left(\frac{d}{2}\right) \zeta(d)}{\pi^{\frac{d}{2}}}, \quad \xi(4)=\frac{\pi^{2}}{90}, \quad \xi(3)=\frac{\zeta(3)}{2 \pi} .
$$

The computation of the Casimir energy of the system yields the same result than for electromagnetism and can be performed in several ways. The result is

$$
E_{0}(0)=-\xi(4) \frac{L_{1} L_{2}}{L_{3}^{3}} .
$$

The partition function can then be directly computed using a quantum statistical approach in a Hilbert space basis that diagonalizes the Hamiltonian. The result is

$$
\begin{aligned}
\ln Z(\beta, \alpha)= & -\beta E_{0}+\xi(3) \frac{L_{1} L_{2}}{\beta^{2}} \\
& +2 \frac{L_{1} L_{2}}{L_{3}^{\frac{3}{2}} \beta^{\frac{1}{2}}} \sum_{n_{3}}^{\prime} \sum_{l \in \mathbb{N}^{*}}\left(\frac{\left|n_{3}\right|}{l}\right)^{\frac{3}{2}} K_{\frac{3}{2}}\left(2 \pi l\left|n_{3}\right| \frac{\beta}{L_{3}}\right) e^{2 \pi i l n_{3} \frac{\alpha}{L_{3}}},
\end{aligned}
$$


where $K_{\frac{3}{2}}\left(2 \pi m \frac{\beta}{L_{3}}\right)$ is a modified Bessel function of the second kind. This result can also be written as

$$
\begin{aligned}
\ln Z(\beta, \alpha)= & \frac{L_{1} L_{2}}{L_{3}^{2}}\left[\xi(4) \frac{\beta}{L_{3}}+\xi(3)\left(\frac{L_{3}}{\beta}\right)^{2}\right. \\
& \left.+2\left(\frac{L_{3}}{\beta}\right)^{\frac{1}{2}} \sum_{n_{3}}^{\prime} \sum_{l \in \mathbb{N}^{*}}\left(\frac{\left|n_{3}\right|}{l}\right)^{\frac{3}{2}} K_{\frac{3}{2}}\left(2 \pi l\left|n_{3}\right| \frac{\beta}{L_{3}}\right) e^{2 \pi i \ln _{3} \frac{\alpha}{L_{3}}}\right] .
\end{aligned}
$$

At low temperature/small distance, $\frac{\beta}{L_{3}} \gg 1$, the leading term in the expansion to the partition function is directly related to the Casimir energy. The leading correction is the contribution of the modes with spatial frequencies $n_{3}=0$ and with normal ordered Hamiltonian. In this context, these are the gravitons that propagate parallel to the plates, which is the sector of the theory explicitly discussed in section 3.3. It is independent of the small dimension $L_{3}$ and coincides with the black body result of electromagnetism in 2 spatial dimensions or with a single massless scalar field in 2 spatial dimensions. On account of the asymptotic expansion

$$
K_{\nu}(z)=\sqrt{\frac{\pi}{2 z}} e^{-z}\left(1+O\left(z^{-1}\right)\right), \quad\left(|\arg z|<\frac{3}{2} \pi\right),
$$

for large $|z|$, all other terms are exponentially suppressed. It follows that in the lowtemperature expansion of the entropy,

$$
S(\beta, \alpha)=\left(1-\beta \partial_{\beta}\right) \ln Z(\beta, \alpha)=3 \xi(3) \frac{L_{1} L_{2}}{\beta^{2}}+\ldots,
$$

the first term in (5.13) proportional to the Casimir energy drops out since it is linear in $\beta$. The dots denote exponentially suppressed terms. The leading contribution now comes from the lower dimensional electromagnetic/scalar field, i.e., the modes with $n_{3}=0$, and scales with the area.

When expressed in terms of inverse temperature and chemical potential, the generating set of transformations of the modular group become

$$
\tau^{\prime}=\tau+1 \Longleftrightarrow\left\{\begin{array}{l}
\beta^{\prime}=\beta \\
\alpha^{\prime}=\alpha+L_{3}
\end{array} \quad \tau^{\prime}=-\frac{1}{\tau} \Longleftrightarrow\left\{\begin{array}{l}
\frac{\beta^{\prime}}{L_{3}}=\frac{L_{3} \beta}{\alpha^{2}+\beta^{2}} \\
\frac{\alpha^{\prime}}{L_{3}}=-\frac{L_{3} \alpha}{\alpha^{2}+\beta^{2}}
\end{array} .\right.\right.
$$

Under the first of these transformations, $\ln Z(\beta, \alpha)$ in (5.13) is manifestly invariant, as required by (5.7) for $c=0, a=b=d=1$. For the second of these transformations, we get

$$
\ln Z\left(\beta^{\prime}, \alpha^{\prime}\right)=\frac{\alpha^{2}+\beta^{2}}{L_{3}^{2}} \ln Z(\beta, \alpha) .
$$

When transposing and using the explicit expression in (5.14) for the l.h.s., this gives

$$
\begin{aligned}
\ln Z(\beta, \alpha)= & \frac{L_{1} L_{2}}{\alpha^{2}+\beta^{2}}\left[\xi(4) \frac{L_{3} \beta}{\alpha^{2}+\beta^{2}}+\xi(3)\left(\frac{\alpha^{2}+\beta^{2}}{L_{3} \beta}\right)^{2}\right. \\
& \left.+2\left(\frac{\alpha^{2}+\beta^{2}}{L_{3} \beta}\right)^{\frac{1}{2}} \sum_{n_{3}}^{\prime} \sum_{l \in \mathbb{N}^{*}}\left(\frac{\left|n_{3}\right|}{l}\right)^{\frac{3}{2}} K_{\frac{3}{2}}\left(2 \pi l\left|n_{3}\right| \frac{L_{3} \beta}{\alpha^{2}+\beta^{2}}\right) e^{-2 \pi i l n_{3} \frac{L_{3} \alpha}{\alpha^{2}+\beta^{2}}}\right]
\end{aligned}
$$


As a consequence, in the high temperature/large distance limit, $\frac{\beta}{L_{3}} \ll 1$, the partition function is given by the first term of the above equation. In the expression of the partition function in terms of the real analytic Eisenstein (5.5), this term comes from the modes with vanishing Matsubara frequencies $n_{4}=0$. For $\alpha=0$, this gives the standard black body result (B.17) when taking into account that the volume of the system is $V=L_{1} L_{2} d$. More generally, the leading contributions are given by the first two terms, while the others are exponentially suppressed. The high temperature/large distance expansion of the entropy is

$$
S(\beta, \alpha)=L_{1} L_{2}\left[L_{3} \xi(4) \frac{4 \beta^{3}}{\left(\alpha^{2}+\beta^{2}\right)^{3}}+\xi(3) \frac{3 \alpha^{2}+\beta^{2}}{\left(L_{3} \beta\right)^{2}}+\ldots\right],
$$

up to exponentially suppressed terms. When $\alpha=0$, this gives the black body result corrected by a temperature-independent term,

$$
S(\beta, 0)=8 \xi(4) \frac{L_{1} L_{2} d}{\beta^{3}}+\frac{\xi(3)}{4} \frac{L_{1} L_{2}}{d^{2}}+\ldots
$$

The leading term is directly related to the Casimir energy of the system, as discussed in the context of the Cardy formula and modular invariance in higher dimensions in [2-4].

\subsection{Complex trigonometric functions from method of images}

Both (5.5) and (5.14) can be shown to be equivalent to a single series in terms of complex trigonometric functions,

$$
\ln \mathcal{Z}(\tau, \bar{\tau})=\frac{\pi^{2} L_{1} L_{2} \mathfrak{I m}(\tau)}{4 L_{3}^{2}}\left[\frac{2}{45}+\sum_{l \in \mathbb{N}^{*}}\left(\frac{i \cot \pi l \tau}{(\pi l \mathfrak{I m}(\tau))^{3}}-\frac{1}{(\pi l \mathfrak{I m}(\tau))^{2} \sin ^{2} \pi l \tau}+\text { c.c. }\right)\right] .
$$

If the chemical potential $\alpha$ vanishes, $\tau=i t=i \frac{\beta}{L_{3}}$, by using $\sin (i x)=i \sinh (x)$ and $\cot (i x)=-i \operatorname{coth}(x)$, we get

$$
\ln \mathrm{Z}(t)=\frac{\pi^{2} L_{1} L_{2} t}{2 L_{3}^{2}}\left[\frac{1}{45}+\sum_{l \in \mathbb{N}^{*}}\left(\frac{\operatorname{coth} \pi l t}{(\pi l t)^{3}}+\frac{1}{(\pi l t)^{2} \sinh ^{2} \pi l t}\right)\right] .
$$

This is the form under which the result usually appears in the Casimir literature. It has originally been derived in [26] by applying a Sommerfeld-Watson transform to the Epstein zeta function (5.8), which in turn had been obtained through the method of images.

\section{Discussion}

As shown by the detailed Hamiltonian analysis, our version of perfectly conducting boundary conditions for massless spin 2 fields are fully consistent with gauge invariance. That the existence of such boundary conditions is non-trivial has been mentioned for instance in [30] (end of section 4). It would be interesting to explore in detail how these boundary conditions relate to those discussed recently in [39].

At the level of the free theory, an open question concerns ADM surface charges for the set-up we are considering. This is relevant in relation to black holes because in the 
Gibbons-Hawking treatment, these charges do play a crucial role. It would be interesting to see whether they can be understood in terms of the particles present in the spectrum, in analogy with what can be done for electric charge in the case of the electromagnetic field between two perfectly conducting plates. The other obvious question is to extend the analysis to more general boundary geometries and to curved backgrounds.

With the boundary conditions that we have chosen, the Casimir force on the "walls" will be the same for gravitons than for photons. Trying to give a meaning to this brings one back to the discussion in the introduction of what type of wall that would be. More generally, in order to distinguish the result for linearized gravity from that for electromagnetism and to begin to discuss potential implications, one needs to consider interactions, and thus bring in Newton's constant, in one way or another. We are looking forward to studying all these questions in the near future.

\section{Acknowledgments}

This work is supported by the F.R.S.-FNRS Belgium through conventions FRFC PDR T.1025.14 and IISN 4.4503.15.

\section{A Photons in a Casimir box revisited}

In this appendix, we re-derive results on the scalar field formulation and modular covariance in the electromagnetic finite temperature Casimir effect [17]. This is done in terms of suitable polarization vectors, so that the construction may be generalized in a straightforward way to the massless spin 2 case.

\section{A.1 Boundary conditions and mode decomposition}

Consider coordinates $x^{i}, i=1,2,3$ in Euclidean space. The electric field is $E^{i}=-\pi^{i}$, the magnetic field is $B^{i}=\epsilon^{i j k} \partial_{j} A_{k}$. The starting point is the first order action

$$
S=\int \mathrm{d} x^{0}\left[\int_{V} \mathrm{~d}^{3} x \partial_{0} A_{i} \pi^{i}-H+\int_{V} \mathrm{~d}^{3} x A_{0} \partial_{i} \pi^{i}\right], \quad H=\frac{1}{2} \int_{V} \mathrm{~d}^{3} x\left(\pi^{i} \pi_{i}+B^{i} B_{i}\right) .
$$

Let $a=1,2, i=a, 3, V=L_{1} L_{2} d$ with $L_{a}$ large, $k_{3}=\frac{\pi n_{3}}{d}, k_{a}=\frac{2 \pi n_{a}}{L_{a}}$ (with no summation over $a$ ). Perfectly conducting boundary conditions on parallel plates at $x^{3}=-\frac{d}{2}$ and $x^{3}=\frac{d}{2}$ require $B^{3}=0$ and $E^{a}=0$ on the plates. Let

$$
\psi_{k}^{H}=\sqrt{\frac{2}{V}} e^{i k_{a} x^{a}} \sin k_{3} x^{3}, \quad \psi_{k_{a}, 0}^{E}=\frac{1}{\sqrt{V}} e^{i k_{a} x^{a}}, \quad \psi_{k}^{E}=\sqrt{\frac{2}{V}} e^{i k_{a} x^{a}} \cos k_{3} x^{3},
$$

and let us use $V^{i}(x)$ for either of the canonically conjugate variables $A^{i}(x)$ or $\pi^{i}(x)$. The boundary conditions are implemented through ${ }^{2}$

$$
V^{a}(x)=i \sum_{n_{a}, n_{3}>0} V_{k}^{a} \psi_{k}^{H}, \quad V^{3}(x)=\sum_{n_{a}, n_{3} \geq 0} V_{k}^{3} \psi_{k}^{E},
$$

where we take $V_{k_{a}, 0}^{a}=0$. Reality and parity conditions are

$V_{k_{a}, k_{3}}^{a}=-V_{-k_{a}, k_{3}}^{* a}, \quad V_{k_{a}, k_{3}}^{3}=V_{-k_{a}, k_{3}}^{* 3}, \quad V_{k_{a}, k_{3}}^{a}=-V_{k_{a},-k_{3}}^{a}, \quad V_{k_{a}, k_{3}}^{3}=V_{k_{a},-k_{3}}^{3}$.

\footnotetext{
${ }^{2}$ The factor $i$ in front of the expansion of $\left(A^{a}, \pi^{a}\right)$ is chosen for later convenience.
} 


\section{A.2 The particle}

The mode at $n_{i}=0, A_{3,0,0}=q, \pi_{0,0}^{3}=p$ is treated separately. It is not affected by the constraints nor by proper gauge transformations. Its Poisson brackets are canonical and its contribution to the Hamiltonian is that of a free particle of unit mass,

$$
H_{n_{i}=0}=\frac{1}{2} p^{2}
$$

\section{A.3 Polarization vectors}

Take now $\alpha=1,2$, and $A=(\alpha, \|)$. In Euclidean momentum space $k^{i}$ (minus the origin) let $k=\sqrt{k_{i} k^{i}}$ and consider an orthonormal frame $e_{A}{ }^{i}(k)$ built out of two vectors normal to $k^{i}$ and one vector parallel to $k^{i}$,

$$
e_{\|}^{i}=\frac{k^{i}}{k}, \quad \epsilon_{j m}^{i} e_{1}^{j} e_{2}^{m}=e_{\|}^{i}, \quad e_{A}{ }^{i} e^{B}{ }_{i}=\delta_{A}^{B}, \quad e_{A}{ }^{i} e^{B}{ }_{j}=\delta_{A}^{B},
$$

so that the decomposition of a vector $v^{i}(k)$ in momentum space in this frame is $V^{i}(k)=$ $V^{A}(k) e_{A}{ }^{i}$ with inverse $V^{A}(k)=V^{i} e^{A}{ }_{i}$.

The non-vanishing Poisson brackets for these modes are read off from the expansion of $\int_{V} \mathrm{~d}^{3} x \partial_{0} A_{i} \pi^{i}$ and given by

$$
\left\{A_{A k}, \pi_{k^{\prime}}^{* B}\right\}=\delta_{A}^{B} \prod_{i=1}^{3} \delta_{n_{i}, n_{i}^{\prime}} .
$$

The contribution to the Hamiltonian from the $n_{3}>0$ modes is given by

$$
H_{n_{3} \neq 0}=\frac{1}{2} \sum_{n_{a}, n_{3}>0}\left(\pi_{k}^{A} \pi_{k A}^{*}+k^{2} A_{k}^{\alpha} A_{k \alpha}^{*}\right) .
$$

Let $k_{\perp}=\sqrt{k_{a} k^{a}}$. The contribution from the $n_{3}=0$ modes is

$$
H_{n_{3}=0}=\frac{1}{2} \sum_{n_{a}}^{\prime}\left(\pi_{k_{a}, 0}^{3} \pi_{k_{a}, 0}^{* 3}+k_{\perp}^{2} A_{k_{a}, 0}^{3} A_{k_{a}, 0}^{* 3}\right)
$$

where the prime means that the mode with $n_{i}=0$ is omitted. In order to implement the constraint $\partial_{i} \pi^{i}=0$ and/or the Coulomb gauge condition $\partial^{i} A_{i}=0$,

$$
\partial_{i} V^{i}=-\sum_{n_{a}, n_{3}>0} k V_{k}^{\|} \psi_{k}^{H}=0 \Longleftrightarrow V_{k}^{\|}=0
$$

Note that $V_{k_{a}, 0}^{a}=0$ implies $V_{k_{a}, 0}^{\|}=0$ and that, in order not to introduce spurious variables, one needs to expand $A_{0}$ as

$$
A_{0}=\sum_{n_{a}, n_{3}>0} A_{k, 0} \psi_{k}^{H} .
$$

By variations with respect to $A_{0}$, respectively $A_{k, 0}$, one may then solve the constraints, or equivalently $\pi_{k}^{\|}=0$ in the action, without the need to impose the Coulomb or any other gauge condition. As a consequence, in the first term in (A.8), one may limit the sum over 
$A$ to one over $\alpha$. The same is true for the kinetic term that gives rise to the canonical Poisson brackets. Because proper gauge transformations with gauge parameters satisfying Dirichlet conditions correspond to arbitrary shifts of $A_{k}^{\|}$, it follows that gauge invariant quantities reduced to the constraint surface do not depend on the variables $A_{k}^{\|}, \pi_{k}^{\|}$.

The electric charge observable is gauge invariant and given by

$$
Q=\int_{x^{3}} \mathrm{~d}^{2} x \pi^{3}=-L_{1} L_{2} \sum_{n_{3}>0} \pi_{0, k_{3}}^{3} \psi_{0, k_{3}}^{E}-\sqrt{\frac{L_{1} L_{2}}{d}} p,
$$

where integration is at fixed $\left.x_{3} \in\right]-\frac{d}{2}, \frac{d}{2}\left[\right.$. Since $\pi_{0, k_{3}}^{3}=\pi_{0, k_{3}}^{\|}$, the observable reduces to the last term only on the constraint surface and is $x_{3}$ independent.

\section{A.4 Adapted polarization vectors}

Consider now the following choice of $e_{\alpha}{ }^{i}$ (see e.g. [38]),

$$
e_{H}^{i}=\frac{1}{k_{\perp}}\left(\begin{array}{c}
k_{2} \\
-k_{1} \\
0
\end{array}\right) \quad e_{E}^{i}=\frac{1}{k_{\perp} k}\left(\begin{array}{c}
k_{1} k_{3} \\
k_{2} k_{3} \\
-k_{\perp}^{2}
\end{array}\right),
$$

so that the components $V^{H}, V^{E}, V^{\|}$are given by

$$
V_{k_{a}, k_{3}}^{H}=\frac{\epsilon^{a b} k_{b}}{k_{\perp}} V_{k_{a}, k_{3}}^{a}, \quad V_{k_{a}, k_{3}}^{E}=\frac{k_{a} k_{3}}{k_{\perp} k} V_{k_{a}, k_{3}}^{a}-\frac{k_{\perp}}{k} V_{k_{a}, k_{3}}^{3}, \quad V_{k_{a}, k_{3}}^{\|}=\frac{k_{i}}{k} V_{k_{a}, k_{3}}^{i},
$$

with inverse relations

$$
V_{k_{a}, k_{3}}^{a}=\frac{\epsilon^{a b} k_{b}}{k_{\perp}} V_{k_{a}, k_{3}}^{H}+\frac{k^{a} k_{3}}{k_{\perp} k} V_{k_{a}, k_{3}}^{E}+k^{a} V_{k_{a}, k_{3}}^{\|}, \quad V_{k_{a}, k_{3}}^{3}=-\frac{k_{\perp}}{k} V_{k_{a}, k_{3}}^{E}+k_{3} V_{k_{a}, k_{3}}^{\|} .
$$

Note in particular that $V_{k_{a}, 0}^{3}=-V_{k_{a}, 0}^{E}$. Reality and parity conditions become

$$
V_{k_{a}, k_{3}}^{H}=V_{-k_{a}, k_{3}}^{* H}, \quad V_{k_{a}, k_{3}}^{E}=V_{-k_{a}, k_{3}}^{* E}, \quad V_{k_{a},-k_{3}}^{H}=-V_{k_{a}, k_{3}}^{H}, \quad V_{k_{a}, k_{3}}^{E}=V_{k_{a},-k_{3}}^{E} .
$$

Let $\lambda=(H, E)$. Oscillator variables are defined as

$$
\begin{aligned}
& a_{k_{a}, k_{3}}^{\lambda}=\sqrt{\frac{k}{2}}\left(A_{k_{a}, k_{3}}^{\lambda}+\frac{i}{k} \pi_{k_{a}, k_{3}}^{\lambda}\right) \\
& A_{k_{a}, k_{3}}^{\lambda}=\frac{1}{\sqrt{2 k}}\left(a_{k_{a}, k_{3}}^{\lambda}+a_{-k_{a}, k_{3}}^{* \lambda}\right), \quad \pi_{k_{a}, k_{3}}^{\lambda}=-i \sqrt{\frac{k}{2}}\left(a_{k_{a}, k_{3}}^{\lambda}-a_{-k_{a}, k_{3}}^{* \lambda}\right)
\end{aligned}
$$

with the understanding that $a_{k_{a}, 0}^{H}=0$. Their non-vanishing Poisson brackets are

$$
\left\{a_{k}^{\lambda}, a_{k^{\prime}}^{* \lambda^{\prime}}\right\}=-i \delta^{\lambda, \lambda^{\prime}} \prod_{i=1}^{3} \delta_{n_{i}, n_{i}^{\prime}} .
$$


In terms of these oscillators, we have

$$
\begin{aligned}
A^{a} & =i \sum_{n_{a}, n_{3}>0}\left[\frac{\epsilon^{a b} k_{b}}{\sqrt{2 k} k_{\perp}}\left(a_{k}^{H} \psi_{k}^{H}-\text { c.c. }\right)+\frac{k^{a} k_{3}}{\sqrt{2 k} k_{\perp} k}\left(a_{k}^{E} \psi_{k}^{H}-\text { c.c. }\right)+k^{a} A_{k}^{\|} \psi_{k}^{H}\right], \\
\pi^{a} & =\sum_{n_{a}, n_{3}>0}\left[\frac{\sqrt{k} \epsilon^{a b} k_{b}}{\sqrt{2} k_{\perp}}\left(a_{k}^{H} \psi_{k}^{H}+\text { c.c. }\right)+\frac{k^{a} k_{3}}{\sqrt{2 k} k_{\perp}}\left(a_{k}^{E} \psi_{k}^{H}+\text { c.c. }\right)+i k^{a} \pi_{k}^{\|} \psi_{k}^{H}\right], \\
A^{3} & =\sum_{n_{a}, n_{3} \geq 0}^{\prime}\left[-\frac{k_{\perp}}{\sqrt{2 k} k}\left(a_{k}^{E} \psi_{k}^{E}+\text { c.c. }\right)+k_{3} A_{k}^{\|} \psi_{k}^{E}\right]+\frac{1}{\sqrt{V}} q, \\
\pi^{3} & =\sum_{n_{a}, n_{3} \geq 0}^{\prime}\left[i \frac{k_{\perp}}{\sqrt{2 k}}\left(a_{k}^{E} \psi_{k}^{E}-\text { c.c. }\right)+k_{3} \pi_{k}^{\|} \psi_{k}^{E}\right]+\frac{1}{\sqrt{V}} p .
\end{aligned}
$$

On the constraint surface, the full Hamiltonian is given by

$$
H=\sum_{\lambda, n_{a}, n_{3} \geq 0}^{\prime} \frac{1}{2}\left(\pi_{k}^{\lambda} \pi_{k}^{* \lambda}+k^{2} A_{k}^{\lambda} A_{k}^{* \lambda}\right)+\frac{1}{2} p^{2}=\sum_{\lambda, n_{a}, n_{3} \geq 0}^{\prime} \frac{k}{2}\left(a_{k}^{\lambda} a_{k}^{* \lambda}+a_{k}^{* \lambda} a_{k}^{\lambda}\right)+\frac{1}{2} p^{2} .
$$

Besides the electric charge operator

$$
Q=-\sqrt{\frac{L_{1} L_{2}}{d}} p
$$

another observable of interest to us is directly related to spin angular momentum of light. In reduced phase space, the latter is given by

$$
J_{i}=\int_{V} \mathrm{~d}^{3} x \epsilon_{i j k} A_{\perp}^{j} \pi_{\perp}^{k}=\int_{V} \mathrm{~d}^{3} x \vec{\pi}_{\perp} \cdot\left(\vec{e}_{i} \times \vec{A}_{\perp}\right),
$$

where $V_{i}^{\perp}$ correspond to the vectors in (A.19) with $V_{k}^{\|}=0=q=p$. When using that

$$
e_{H}^{i} e_{E}^{j}-e_{E}^{i} e_{H}^{j}=\epsilon^{i j l} \frac{k_{l}}{k}
$$

it follows that, in terms of modes,

$$
J_{3}=\sum_{n_{a}, n_{3}>0} \frac{k_{3}}{k}\left(A_{k}^{H} \pi_{k}^{* E}-A_{k}^{E} \pi_{k}^{* H}\right)=\sum_{n_{a}, n_{3}>0} \frac{i k_{3}}{k}\left(a_{k}^{* H} a_{k}^{E}-a_{k}^{* E} a_{k}^{H}\right),
$$

More precisely, because we are interested in a modular covariant partition function, we are interested in the observable

$$
P_{3}^{S}=-\int_{V} \mathrm{~d}^{3} x \partial_{3} B^{i} \frac{1}{\sqrt{-\triangle}} \pi_{i}
$$

whose mode expansion can be shown to differ from that of $J_{3}$ by multiplying each term in momentum space by $k$,

$$
P_{3}^{S}=\sum_{n_{a}, n_{3}>0} k_{3}\left(A_{k}^{H} \pi_{k}^{* E}-A_{k}^{E} \pi_{k}^{* H}\right)=\sum_{n_{a}, n_{3}>0} i k_{3}\left(a_{k}^{* H} a_{k}^{E}-a_{k}^{* E} a_{k}^{H}\right)
$$




\section{A.5 Bromwich-Borgnis fields}

Consider now the real fields

$$
\begin{aligned}
\phi^{H} & =\sum_{n_{a}, n_{3}>0} \frac{1}{\sqrt{2 k} k_{\perp}}\left(a_{k}^{H} \psi_{k}^{H}+\text { c.c. }\right), & \pi^{H} & =-i \sum_{n_{a}, n_{3}>0} \frac{\sqrt{k}}{\sqrt{2} k_{\perp}}\left(a_{k}^{H} \psi_{k}^{H}-\text { c.c. }\right) \\
\phi^{E} & =-\sum_{n_{a}, n_{3} \geq 0}^{\prime} \frac{1}{\sqrt{2 k} k k_{\perp}}\left(a_{k}^{E} \psi_{k}^{E}+\text { c.c. }\right), & \pi^{E} & =i \sum_{n_{a}, n_{3} \geq 0}^{\prime} \frac{1}{\sqrt{2 k} k_{\perp}}\left(a_{k}^{E} \psi_{k}^{E}-\text { c.c. }\right) \\
\phi^{G} & =\sum_{n_{a}, n_{3}>0} \frac{1}{2}\left(A_{k}^{\|} \psi_{k}^{H}+\text { c.c. }\right), & \pi^{G} & =\sum_{n_{a}, n_{3}>0} \frac{1}{2}\left(\pi_{k}^{\|} \psi_{k}^{H}+\text { c.c. }\right) .
\end{aligned}
$$

Let $\Lambda=(H, E, G)$ and $\varphi^{\Lambda}$ stand for either $\phi^{\Lambda}$ or $\pi^{\Lambda}$. The fields $\varphi^{H}, \varphi^{G}$ satisfy Dirichlet conditions while $\varphi^{E}$ satisfies Neumann conditions. In these terms,

$$
\begin{aligned}
A^{a}=\epsilon^{a b} \partial_{b} \phi^{H}+\partial^{a} \partial_{3} \phi^{E}+\partial^{a} \phi^{G}, & A^{3}=\left(-\triangle+\partial_{3}^{2}\right) \phi^{E}+\partial^{3} \phi^{G}+\frac{1}{\sqrt{V}} q, \\
\pi^{a}=\epsilon^{a b} \partial_{b} \pi^{H}+\partial^{a} \partial_{3} \pi^{E}+\partial^{a} \pi^{G}, & \pi^{3}=\left(-\triangle+\partial_{3}^{2}\right) \pi^{E}+\partial^{3} \pi^{G}+\frac{1}{\sqrt{V}} p, \\
B^{a}=\epsilon^{a b} \partial_{b}(-\triangle) \phi^{E}+\partial^{a} \partial_{3} \phi^{H}, & B^{3}=\left(-\triangle+\partial_{3}^{2}\right) \phi^{H} .
\end{aligned}
$$

On the constraint surface where $\pi^{G}=0$, one recovers the construction of [40,41] (see also [42] section 32 and $[1,36,43]$ for related more modern discussions).

\section{A.6 Single scalar field formulation}

In order to streamline the computation of the partition function and to discuss modular properties, it is useful to go one step further and introduce a formulation with a single scalar field on $z \in[-d, d]$ with periodic boundary conditions [17].

The variables defined by

$$
\begin{aligned}
\phi_{k_{a}, k_{3}} & =\frac{A_{k_{a}, k_{3}}^{E}-i A_{k_{a}, k_{3}}^{H},}{\sqrt{2}}, & \pi_{k_{a}, k_{3}} & =\frac{\pi_{k_{a}, k_{3}}^{E}-i \pi_{k_{a}, k_{3}}^{H}}{\sqrt{2}}, \\
\phi_{k_{a}, 0} & =A_{k_{a}, 0}^{E}, & \pi_{k_{a}, 0} & =\pi_{k_{a}, 0}^{E},
\end{aligned}
$$

satisfy the reality conditions

$$
\phi_{-k_{a}, 0}^{*}=\phi_{k_{a}, 0}, \quad \phi_{-k_{a},-k_{3}}^{*}=\phi_{k_{a}, k_{3}}, \quad \pi_{-k_{a}, 0}^{*}=\pi_{k_{a}, 0}, \quad \pi_{-k_{a},-k_{3}}^{*}=\pi_{k_{a}, k_{3}} .
$$

The first expression for the Hamiltonian in (A.20) can then be written as

$$
H=\sum_{n_{i}} \frac{1}{2}\left(\pi_{k} \pi_{k}^{*}+k^{2} \phi_{k} \phi_{k}^{*}\right)
$$

with the understanding that $\pi_{0,0}=p, \phi_{0,0}=q$ and the sum goes over all $n_{i} \in \mathbb{Z}$. In terms of appropriate oscillators, defined for $n_{i} \neq 0$,

$$
a_{k_{a}, k_{3}}=\sqrt{\frac{k}{2}}\left(\phi_{k_{a}, k_{3}}+\frac{i}{k} \pi_{k_{a}, k_{3}}\right)=\left\{\begin{array}{ll}
\frac{a_{k_{a}, k_{3}}^{E}-i a_{k_{a}, k_{3}}^{H},}{\sqrt{2}}, & n_{3} \neq 0, \\
a_{k_{a}, 0}^{E}, & n_{3} \neq 0
\end{array},\right.
$$


the Hamiltonian becomes

$$
H=\sum_{n_{i}}^{\prime} \frac{k}{2}\left(a_{k}^{*} a_{k}+a_{k} a_{k}^{*}\right)+\frac{1}{2} p^{2} .
$$

The above are the mode decomposition, oscillators and Hamiltonian of a single real scalar field $\phi$ and its momentum $\pi$ in a volume $V^{\prime}=L_{1} L_{2} L_{3}$ where $L_{3}=2 d$ with periodic boundary conditions in all directions and, in particular, with periodicity $2 d$ in the $x^{3}$ direction.

$$
\begin{aligned}
& \phi=\frac{1}{\sqrt{V^{\prime}}} \sum_{n_{i}} e^{i k_{j} x^{j}} \phi_{k_{i}}=\frac{1}{\sqrt{V^{\prime}}}\left(\sum_{n_{i}}^{\prime} \frac{1}{\sqrt{2 k}}\left[a_{k_{i}} e^{i k_{j} x^{j}}+a_{k_{i}}^{*} e^{-i k_{j} x^{j}}\right]+q\right), \\
& \pi=\frac{1}{\sqrt{V^{\prime}}} \sum_{n_{i}} e^{i k_{j} x^{j}} \pi_{k_{i}}=\frac{1}{\sqrt{V^{\prime}}}\left(-i \sum_{n_{i}}^{\prime} \sqrt{\frac{k}{2}}\left[a_{k_{i}} e^{i k_{j} x^{j}}-a_{k_{i}}^{*} e^{-i k_{j} x^{j}}\right]+p\right),
\end{aligned}
$$

for which the Hamiltonian is

$$
H=\frac{1}{2} \int_{V^{\prime}} \mathrm{d}^{3} x\left(\pi^{2}+\partial_{i} \phi \partial^{i} \phi\right)
$$

By design of $P_{3}^{S}$ in (A.25), the mode expansion of linear momentum along $x^{3}$ of the scalar field,

$$
P_{3}^{S}=-\int_{V^{\prime}} \mathrm{d}^{3} x \pi \partial_{3} \phi=\sum_{n_{i}} k_{3} a_{k}^{*} a_{k},
$$

can be easily shown to agree with (A.26) when using (A.32).

\section{A.7 Partition function of photons}

For the computation of the partition function for photons in a Casimir box,

$$
Z(\beta, \alpha, \mu)=\operatorname{Tr} e^{-\beta(\hat{H}-\mu \hat{Q})+\alpha \hat{P}_{3}^{S}},
$$

one may now use the scalar field formulation to show that

$$
\ln Z(\beta, \alpha, \mu)=\frac{L_{1} L_{2}}{L_{3}} \beta \mu^{2}+\ln \mathcal{Z}(\tau, \bar{\tau}) .
$$

The first terms comes from the particle and reproduces the Gibbons-Hawking contribution to the planar capacitor $[15,16]$. It is a classical contribution on top of the standard contribution corresponding to the Casimir energy at zero temperature. The second term corresponds to the modular covariant partition function of a massless scalar field on $\mathbb{S}_{2 d}^{1} \times$ $\mathbb{R}^{2}$. Several equivalent expressions are given in the main text in section 5 .

\section{B Linearized gravity in empty space}

\section{B.1 Hamiltonian formulation of Pauli-Fierz theory}

The Hamiltonian formulation of massless spin 2 fields may be obtained by linearizing the $\mathrm{ADM}$ formulation of full general relativity around flat space, with the result

$$
S_{\mathrm{PF}}\left[h_{i j}, \pi^{i j}, n_{i}, n\right]=\int \mathrm{d} x^{0}\left[\int \mathrm{d}^{3} x\left(\pi^{i j} \partial_{0} h_{i j}-n^{i} \mathcal{H}_{i}-n \mathcal{H}_{\perp}\right)-H_{\mathrm{PF}}\right],
$$


where

$$
\begin{aligned}
H_{\mathrm{PF}}\left[h_{i j}, \pi^{i j}\right]= & \int \mathrm{d}^{3} x\left(\pi^{i j} \pi_{i j}-\frac{1}{2} \pi^{2}+\frac{1}{4} \partial^{l} h^{i j} \partial_{l} h_{i j}-\right. \\
& \left.-\frac{1}{2} \partial_{i} h^{i j} \partial^{l} h_{l j}+\frac{1}{2} \partial^{i} h \partial^{j} h_{i j}-\frac{1}{4} \partial^{i} h \partial_{i} h\right),
\end{aligned}
$$

and

$$
\mathcal{H}_{i}=-2 \partial^{j} \pi_{i j}, \quad \mathcal{H}_{\perp}=\Delta h-\partial^{i} \partial^{j} h_{i j} .
$$

Indices are lowered and raised with the flat space metric $\delta_{i j}$ and its inverse, $h=h^{i}{ }_{i}, \pi=\pi^{i}{ }_{i}$ and $\Delta=\partial_{i} \partial^{i}$ is the Laplacian in flat space. The linearized 4 metric is reconstructed using $h_{00}=-2 n$ and $h_{0 i}=n_{i}$.

One may now discuss the decomposition of symmetric rank two tensors $\phi_{i j}, \pi^{i j}$ into transverse trace-less, trace and longitudinal parts [44, 45] either in real space or in reciprocal space. For the purpose of computing a partition function in physical Fock space, we will do the latter. In what follows, we thus consider the Fourier transforms of the fields

$$
\phi(k)=\frac{1}{(2 \pi)^{\frac{3}{2}}} \int \mathrm{d}^{3} x e^{-i k_{j} x^{j}} \phi(x) .
$$

\section{B.2 Polarization tensors}

Let $e_{A}{ }^{i}, A=(\alpha, \|), \alpha=1,2$ be the orthonormal frame for vectors as in (A.6). Let $\Xi=(T T s, T, L T \alpha, L L)$, with $s=(+, \times)$. When $k_{i} \neq 0$, an orthonormal basis for symmetric tensors $e_{\Xi}^{i j}$ is constructed as follows:

$$
\begin{aligned}
& e_{T T+}{ }^{i j}=\frac{1}{\sqrt{2}}\left(e_{1}^{i} e_{1}^{j}-e_{2}{ }^{i} e_{2}^{j}\right), \quad e_{T T \times}{ }^{i j}=\frac{1}{\sqrt{2}}\left(e_{1}{ }^{i} e_{2}{ }^{j}+e_{2}{ }^{i} e_{1}^{j}\right), \\
& k_{i} e_{T T s}^{i j}=0 \text {, } \\
& \delta_{i j} e_{T T s}^{i j}=0 \text {, } \\
& e_{T}^{i j}=\frac{1}{\sqrt{2}}\left(\delta^{i j}-e_{\|}^{i} e_{\|}^{j}\right), \quad k_{i} e_{T}^{i j}=0, \quad \delta_{i j} e_{T}^{i j}=\sqrt{2}, \\
& e_{L T \alpha}{ }^{i j}=\frac{1}{\sqrt{2}}\left(e_{\|}{ }^{i} e_{\alpha}{ }^{j}+e_{\alpha}{ }^{i} e_{\|}^{j}\right), \quad k_{i} e_{L T \alpha}{ }^{i j}=\frac{k}{\sqrt{2}} e_{\alpha}{ }^{j}, \quad \delta_{i j} e_{L T \alpha}{ }^{i j}=0, \\
& e_{L L}{ }^{i j}=e_{\|}{ }^{i} e_{\|}{ }^{j}, \quad k_{i} e_{L L}{ }^{i j}=k^{j}, \quad \delta_{i j} e_{L L}{ }^{i j}=1 .
\end{aligned}
$$

This basis is orthonormal,

$$
e_{\Xi}^{i j} e^{\Gamma}{ }_{i j}=\delta_{\Xi}^{\Gamma}, \quad e_{\Xi}^{i j} e^{\Xi}{ }_{m n}=\frac{1}{2}\left(\delta_{m}^{i} \delta_{n}^{j}+\delta_{n}^{i} \delta_{m}^{j}\right),
$$

so that the Fourier components of $h_{m n}, \pi^{m n}$ may be decomposed as

$$
h_{i j}=h_{\Xi} e^{\Xi}{ }_{i j}, \quad h_{\Xi}=h_{i j} e_{\Xi} \Xi^{i j}, \quad \pi^{i j}=\pi^{\Xi} e_{\Xi}{ }^{i j}, \quad \pi^{\Xi}=\pi^{i j} e_{i j}{ }^{\Xi} .
$$

With this decomposition, the kinetic term becomes

$$
\int \mathrm{d}^{3} x \dot{h}_{i j} \pi^{i j}=\int \mathrm{d}^{3} k \dot{h}_{\Xi} \pi^{* \Xi},
$$


with the usual reality conditions, so that the non-vanishing Poisson are

$$
\left\{h_{\Xi}(k), \pi^{* \Gamma}\left(k^{\prime}\right)\right\}=\delta \delta_{\Xi}^{\Gamma} \delta^{3}\left(k, k^{\prime}\right) .
$$

Decomposing the Fourier components of the shift as $n^{i}(k)=n^{A}(k) e_{A}^{i}$, the constraints in the action principle become

$$
\int \mathrm{d}^{3} x\left(n^{i} \mathcal{H}_{i}+n \mathcal{H}\right)=\int \mathrm{d}^{3} k(2 i k)\left(n^{\|} \pi_{L L}^{*}+n^{\alpha} \frac{1}{\sqrt{2}} \pi_{L T \alpha}^{*}-n k^{2} \sqrt{2} h_{T}^{*}\right) .
$$

The constraint surface is thus determined by $\pi^{L L}=0=\pi^{L T \alpha}=h_{T}$. On the constraint surface, the first order action reduces to

$$
\begin{aligned}
& S_{\mathrm{PF}} \approx \int \mathrm{d} x^{0}\left[\int \mathrm{d}^{3} k \partial_{0} h_{T T s} \pi^{* T T s}-H_{\mathrm{PF}}\right], \\
& H_{\mathrm{PF}} \approx \int \mathrm{d}^{3} k\left(\pi_{T T s} \pi^{* T T s}+\frac{1}{4} \pi_{T T s} \pi^{* T T s}\right),
\end{aligned}
$$

without the need to fix a specific gauge.

Defining the oscillator variables,

$$
a_{T T s}=\frac{1}{2} \sqrt{k} h_{T T s}+i \frac{\pi^{T T s}}{\sqrt{k}},
$$

up to integrations by parts in $\partial_{0}$, the first order action becomes

$$
\begin{aligned}
S_{\mathrm{PF}} & \approx \int \mathrm{d} x^{0}\left[\int \mathrm{d}^{3} k \frac{1}{2 i}\left(\partial_{0} a_{T T s}^{*} a^{T T s}-a_{T T s}^{*} \partial_{0} a^{T T s}\right)-H_{\mathrm{PF}}\right], \\
H_{\mathrm{PF}} & \approx \int \mathrm{d}^{3} k k a_{T T s}^{*} a^{T T s} .
\end{aligned}
$$

In the large volume limit, the partition function $Z(\beta)=\operatorname{Tr} e^{-\beta: \hat{H}_{\mathrm{PF}}}$, involving the normal ordered Hamiltonian, is then computed as usual by first putting the system in a box, for instance with periodic boundary conditions. This box is subsequently taken to be large so that one may replace sums by integrals, up to a volume factor. This yields the standard black body result,

$$
\ln Z(\beta)=2 \frac{\pi^{2}}{90} \frac{V}{\beta^{3}}
$$

\section{Generalized curl and projection operators on modes}

Let us consider

$$
\begin{aligned}
\left(\hat{\mathcal{O}} T_{k}\right)^{i j} & =k\left(\overrightarrow{e_{\|}} \times T_{k}\right)^{i j}=\frac{k}{2}\left(\epsilon_{l m}^{i} e_{\|}{ }^{l} T_{k}{ }^{m j}+\epsilon_{l m}^{j} e_{\|}{ }^{l} T_{k}{ }^{i m}\right), \\
\left(\hat{\mathcal{O}} e_{\Xi}\right)^{i j} T_{k}^{\Xi} & =k\left(e_{T T \times}{ }^{i j} T_{k}^{T T+}-e_{T T+}{ }^{i j} T_{k}^{T T \times}+\frac{1}{2} e_{L T E}{ }^{i j} T_{k}^{L T H}-\frac{1}{2} e_{L T H}{ }^{i j} T_{k}^{L T E}\right),
\end{aligned}
$$

and

$$
\begin{aligned}
\left(\hat{\mathcal{P}} T_{k}\right)^{i j} & =k^{2}\left(T_{k}^{i j}-e_{i}{ }^{\|} e_{l}{ }^{\|} T_{k j}{ }^{l}-e_{j}{ }^{\|} e_{l}{ }_{l} T_{k i}{ }^{l}\right), \\
\left(\hat{\mathcal{P}} e_{\Xi}\right)^{i j} T_{k}^{\Xi} & =k^{2}\left(e_{T T+}{ }^{i j} T_{k}^{T T+}+e_{T T \times}{ }^{i j} T_{k}^{T T \times}+e_{T}{ }^{i j} T_{k}^{T}-e_{L L}{ }^{i j} T_{k}^{L L}\right) .
\end{aligned}
$$


In terms of the mode expansion for the Casimir box, the actions of $\mathcal{O}$ in (4.8) and $\mathcal{P}$ in (4.9) are explicitly given by

$$
\begin{aligned}
& (\mathcal{O} T)^{a b}=\sum_{n_{a}, n_{3} \geq 0}^{\prime}\left(\hat{\mathcal{O}} T_{k}\right)^{a b} \psi_{k}^{E}=\sum_{n_{a}, n_{3} \geq 0}^{\prime}\left(\hat{\mathcal{O}} e_{\Xi}\right)^{a b} T_{k}^{\Xi} \psi_{k}^{E}, \\
& (\mathcal{O} T)^{a 3}=\sum_{n_{a}, n_{3}>0} i\left(\hat{\mathcal{O}} T_{k}\right)^{a 3} \psi_{k}^{H}=\sum_{n_{a}, n_{3}>0} i\left(\hat{\mathcal{O}} e_{\Xi}\right)^{a 3} T_{k}^{\Xi} \psi_{k}^{H}, \\
& (\mathcal{O} T)^{33}=\sum_{n_{a}, n_{3} \geq 0}^{\prime}\left(\hat{\mathcal{O}} T_{k}\right)^{33} \psi_{k}^{E}=\sum_{n_{a}, n_{3} \geq 0}^{\prime}\left(\hat{\mathcal{O}} e_{\Xi}\right)^{33} T_{k}^{\Xi} \psi_{k}^{E},
\end{aligned}
$$

and

$$
\begin{aligned}
(\mathcal{P} T)^{a b} & =\sum_{n_{a}, n_{3}>0}\left(\hat{\mathcal{P}} T_{k}\right)^{a b} \psi_{k}^{H}=\sum_{n_{a}, n_{3}>0}\left(\hat{\mathcal{P}} e_{\Xi}\right)^{i j} T_{k}^{\Xi} \psi_{k}^{H}, \\
(\mathcal{P} T)^{a 3} & =\sum_{n_{a}, n_{3} \geq 0}^{\prime}(-i)\left(\hat{\mathcal{P}} T_{k}\right)^{a 3} \psi_{k}^{E}=\sum_{n_{a}, n_{3} \geq 0}^{\prime}(-i)\left(\hat{\mathcal{P}} e_{\Xi}\right)^{a 3} T_{k}^{\Xi} \psi_{k}^{E}, \\
(\mathcal{P} T)^{33} & =\sum_{n_{a}, n_{3}>0}\left(\hat{\mathcal{P}} T_{k}\right)^{33} \psi_{k}^{H}=\sum_{n_{a}, n_{3}>0}\left(\hat{\mathcal{P}} e_{\Xi}\right)^{33} T_{k}^{\Xi} \psi_{k}^{H} .
\end{aligned}
$$

Open Access. This article is distributed under the terms of the Creative Commons Attribution License (CC-BY 4.0), which permits any use, distribution and reproduction in any medium, provided the original author(s) and source are credited.

\section{References}

[1] B.S. DeWitt, Quantum Field Theory in Curved Space-Time, Phys. Rept. 19 (1975) 295 [INSPIRE].

[2] A. Cappelli and A. Coste, On the Stress Tensor of Conformal Field Theories in Higher Dimensions, Nucl. Phys. B 314 (1989) 707 [inSPIRE].

[3] J.L. Cardy, Operator content and modular properties of higher dimensional conformal field theories, Nucl. Phys. B 366 (1991) 403 [InSPIRE].

[4] E. Shaghoulian, Modular forms and a generalized Cardy formula in higher dimensions, Phys. Rev. D 93 (2016) 126005 [arXiv:1508.02728] [inSPIRE].

[5] L. Smolin, The thermodynamics of gravitational radiation, Gen. Rel. Grav. 16 (1984) 205.

[6] D. Garfinkle and R.M. Wald, on the possibility of a box for holding gravitational radiation in thermal equilibrium, Gen. Rel. Grav. 17 (1985) 461 [INSPIRE].

[7] J. Dell, On the impossibility of a box for holding gravitational radiation in thermal equilibrium, Gen. Rel. Grav. 19 (1987) 171 [inSPIRE].

[8] T. Padmanabhan and T.P. Singh, A Note on the thermodynamics of gravitational radiation, Class. Quant. Grav. 20 (2003) 4419 [gr-qc/0305030] [inSPIRE].

[9] J.Q. Quach, Gravitational Casimir effect, Phys. Rev. Lett. 114 (2015) 081104 [Erratum ibid. 118 (2017) 139901] [arXiv: 1502.07429] [INSPIRE]. 
[10] S. Viaggiu, Statistical mechanics of gravitons in a box and the black hole entropy, Physica A 473 (2017) 412 [arXiv: 1701.06193] [INSPIRE].

[11] H.B. Casimir, On the attraction between two perfectly conducting plates, Kon. Ned. Akad. Wetensch. Proc. 51 (1948) 793.

[12] T.H. Boyer, Quantum electromagnetic zero point energy of a conducting spherical shell and the Casimir model for a charged particle, Phys. Rev. 174 (1968) 1764 [INSPIRE].

[13] T.H. Boyer, Concerning the Zeros of Some Functions Related to Bessel Functions, J. Math. Phys. 10 (1969) 1729.

[14] R.H. Brandenberger, T. Prokopec and V.F. Mukhanov, The Entropy of the gravitational field, Phys. Rev. D 48 (1993) 2443 [gr-qc/9208009] [InSPIRE].

[15] G. Barnich, Black hole entropy from nonproper gauge degrees of freedom: The charged vacuum capacitor, Phys. Rev. D 99 (2019) 026007 [arXiv: 1806.00549] [INSPIRE].

[16] G. Barnich and M. Bonte, Soft degrees of freedom, Gibbons-Hawking contribution and entropy from Casimir effect, arXiv:1912.12698 [INSPIRE].

[17] F. Alessio and G. Barnich, Modular invariance in finite temperature Casimir effect, JHEP 10 (2020) 134 [arXiv:2007.13334] [INSPIRE].

[18] F. Alessio, G. Barnich, M. Bonte and A. Kleinschmidt, Notes on scalar field partition functions and Eisenstein series, in preparation.

[19] M. Henneaux and C. Teitelboim, Quantization of Gauge Systems, Princeton University Press, Princeton U.S.A. (1992).

[20] S. Deser and C. Teitelboim, Duality Transformations of Abelian and Nonabelian Gauge Fields, Phys. Rev. D 13 (1976) 1592 [InSPIRE].

[21] M. Henneaux and C. Teitelboim, Duality in linearized gravity, Phys. Rev. D 71 (2005) 024018 [gr-qc/0408101] [INSPIRE].

[22] S. Deser and D. Seminara, Duality invariance of all free bosonic and fermionic gauge fields, Phys. Lett. B 607 (2005) 317 [hep-th/0411169] [INSPIRE].

[23] S. Deser and D. Seminara, Free spin 2 duality invariance cannot be extended to GR, Phys. Rev. D 71 (2005) 081502 [hep-th/0503030] [INSPIRE].

[24] G. Barnich and C. Troessaert, Manifest spin 2 duality with electric and magnetic sources, JHEP 01 (2009) 030 [arXiv:0812.0552] [INSPIRE].

[25] J. Mehra, Temperature correction to the Casimir effect, Physica 37 (1967) 145 [INSPIRE].

[26] L.S. Brown and G.J. Maclay, Vacuum stress between conducting plates: An Image solution, Phys. Rev. 184 (1969) 1272 [inSPIRE].

[27] J.S. Dowker and R. Critchley, Covariant Casimir calculations, J. Phys. A 9 (1976) 535.

[28] R. Balian and B. Duplantier, Electromagnetic Waves Near Perfect Conductors. 2. Casimir Effect, Annals Phys. 112 (1978) 165 [INSPIRE].

[29] S.I. Tadaki and S. Takagi, Casimir effect at finite temperature, Prog. Theor. Phys. 75 (1986) 262 [INSPIRE].

[30] J. Ambjørn and S. Wolfram, Properties of the Vacuum. 1. Mechanical and Thermodynamic, Annals Phys. 147 (1983) 1 [INSPIRE]. 
[31] J. Ambjørn and S. Wolfram, Properties of the Vacuum. 2. Electrodynamic, Annals Phys. 147 (1983) 33 [INSPIRE].

[32] G. Plunien, B. Müller and W. Greiner, Casimir energy at finite temperature, Physica A $\mathbf{1 4 5}$ (1987) 202 [INSPIRE].

[33] C.A. Lütken and F. Ravndal, A Symmetry in the Finite Temperature Casimir Effect, J. Phys. A 21 (1988) L793 [INSPIRE].

[34] L.H. Ford, Spectrum of the Casimir Effect, Phys. Rev. D 38 (1988) 528 [inSPIRE].

[35] F. Ravndal and D. Tollefsen, Temperature inversion symmetry in the Casimir effect, Phys. Rev. D 40 (1989) 4191 [INSPIRE].

[36] G. Plunien, B. Müller and W. Greiner, The Casimir Effect, Phys. Rept. 134 (1986) 87 [INSPIRE].

[37] V.V. Nesterenko, G. Lambiase and G. Scarpetta, Calculation of the Casimir energy at zero and finite temperature: Some recent results, Riv. Nuovo Cim. 27 (2004) 1 [hep-th/0503100] [INSPIRE].

[38] M. Bordag, G.L. Klimchitskaya, U. Mohideen and V.M. Mostepanenko, Advances in the Casimir effect, Int. Ser. Monogr. Phys. 145 (2009) 1.

[39] E. Witten, A Note On Boundary Conditions In Euclidean Gravity, arXiv:1805.11559 [INSPIRE].

[40] T. Bromwich, X. Electromagnetic waves, Lond. Edinb. Dubl. Philos. Mag. J. Sci. 38 (1919) 143.

[41] F. Borgnis, Elektromagnetische Eingenschwingungen dielektrischer Räume, Ann. Phys. 427 (1939) 359.

[42] M. Phillips, Classical electrodynamics, in Encyclopedia of Physics. Vol. IV: Principles of Electrodynamics and Relativity, Springer, Heidelberg Germany (1962).

[43] D. Deutsch and P. Candelas, Boundary Effects in Quantum Field Theory, Phys. Rev. D 20 (1979) 3063 [INSPIRE].

[44] R. Arnowitt, S. Deser and C. Misner, The Dynamics of General Relativity, in Gravitation, an Introduction to Current Research, Wiley, New York U.S.A. (1962), Chapter 7, pg. 227.

[45] S. Deser, Covariant decomposition of symmetric tensors and the gravitational Cauchy problem, Ann. Henri Poincaré 7 (1967) 149. 